\title{
Structural and functional contributions of conducting tissues to genotypic and environmental variations of tomato fruit mass
}

\author{
Jeanne Simon ${ }^{1}$, Maïda Cardoso ${ }^{2}$, Béatrice Brunel ${ }^{1}$, Eric Alibert ${ }^{2}$, Christelle Baptiste ${ }^{3}$, Marc \\ Lartaud $^{3}$, Jean-Luc Verdeil ${ }^{3}$, Gilles Vercambre ${ }^{1}$, Christophe Goze-Bac ${ }^{2}$, and Nadia Bertin ${ }^{1}$ \\ ${ }^{1}$ INRAE \\ ${ }^{2}$ Université de Montpellier \\ ${ }^{3}$ CIRAD Centre de Montpellier
}

May 8, 2020

\begin{abstract}
Conductive tissues are main routes of resource transport, that are crucial for the growth of fleshy fruit. Yet, very few quantitative data of xylem and phloem areas are available and their variabilities are unknown. This study aimed at better understanding and quantifying the structural and functional properties of the conductive tissues in tomato pedicel. 11 contrasting genotypes were described and the impact of water deficit was studied depending on stress intensity and stage of application. In parallel, MRI was used to assess the proportion and size of active xylem vessels in the stem. Results were implemented in a Virtual Fruit model to assess the potential contribution of pedicel conductive tissues in the variability of fruit fresh and dry masses. On their whole, results suggested that variations in the properties of conducting tissues are involved in the genotypic and environmental variations of fruit mass. The study also highlights the interest to combine methods and to integrate knowledge to better understand plant functioning, and finally to improve plant models. Flow-MRI was shown to be an easy non-destructive method to measure the functional properties of conducting tissues such as the proportion of active vessels and their diameter.
\end{abstract}

\section{KEYWORDS}

Solanum lycopersicum, xylem, phloem, histology, water deficit, genotypic variability, Virtual Fruit model, flow-MRI

\section{ACKNOWLEDGEMENTS}

This work was carried out thanks to the support of the «Investissements d'avenir» program (Labex Agro:ANR-10-LABX-0001-01), under the frame of I-SITE MUSE (ANR-16-IDEX-0006) and thanks to the support of the department AgroEnv INRAE. We thank Guillaume Garcia and Thibault Crouzet for their contributions.

Structural and functional contributions of conducting tissues to genotypic and environmental variations of tomato fruit mass

Jeanne Simon ${ }^{1,2^{*}}$, Maïda Cardoso ${ }^{3}$, Béatrice Brunel $^{1}$, Eric Alibert ${ }^{2}$, Christelle Baptiste ${ }^{4}$, Marc Lartaud $^{4}$, Jean-Luc Verdeil ${ }^{4}$, Gilles Vercambre ${ }^{1}$, Christophe Goze-Bac ${ }^{2}$, Nadia Bertin ${ }^{1}$

${ }^{1}$ INRAE UR1115 Plantes et Systèmes de culture Horticoles - Site Agroparc - 84914, Avignon, France, ${ }^{2}$ Université Montpellier-CNRS, Laboratoire Charles Coulomb UMR 5221, F-34095, Montpellier, France, ${ }^{3}$ Université Montpellier, BNIF Imaging facility, F-34095, Montpellier, France; ${ }^{4}$ UMR AGAP, CIRAD, Montpellier, France

* Corresponding author 


\begin{abstract}
Conductive tissues are main routes of resource transport that are crucial for the growth of fleshy fruit. Yet, very few quantitative data of xylem and phloem areas are available and their variabilities are unknown. This study aimed at better understanding and quantifying the structural and functional properties of the conductive tissues in tomato pedicel. 11 contrasting genotypes were described and the impact of water deficit was studied depending on stress intensity and stage of application. In parallel, MRI was used to assess the proportion and size of active xylem vessels in the stem. Results were implemented in a Virtual Fruit model to assess the potential contribution of pedicel conductive tissues in the variability of fruit fresh and dry masses. On their whole, results suggested that variations in the properties of conducting tissues are involved in the genotypic and environmental variations of fruit mass. The study also highlights the interest to combine methods and to integrate knowledge to better understand plant functioning, and finally to improve plant models. Flow-MRI was shown to be an easy non-destructive method to measure the functional properties of conducting tissues such as the proportion of active vessels and their diameter.
\end{abstract}

Keywords: Solanum lycopersicum, xylem, phloem, histology, water deficit, genotypic variability, Virtual Fruit model, flow-MRI

\title{
1 - INTRODUCTION
}

Growth and quality of fleshy fruits, such as tomato, are mainly determined by water and nutrient fluxes from source organs (leaves, roots) to sink organs (fruit, roots, apex...). Resources are transported through the xylem vessels and through the phloem sieve elements that drive respectively, the transport of water and minerals from roots to leaves and fruits, and the translocation of sugar and amino acids from leaves to sink organs (Ho, 1996). Conductive tissues also transport molecules implicated in the signalization on long distances and these molecules play an important role in responses to stress (Lough and Lucas 2006 ; Spiegelman et al., 2015). Many studies have investigated variations in fruit growth, by measuring phloem and xylem fluxes in response to environmental or genetic factors, using destructive or indirect methods. For tomato, isotopic labeling, balance method, heat girdling or MRI have allowed to quantify the xylem and phloem contributions to fruit growth (Ho et al., 1987 ; Guichard et al., 2005 ; Van Bel 1990 ; Windt et al., 2006). It was estimated that 80 to $90 \%$ of water imported by tomato fruit is transported by the phloem, and the remaining 10 to $20 \%$ is transported by the xylem (Ho et al., 1987 ; Guichard et al., 2005). However, using MRI, a direct non-destructive method, Windt et al. (2009) suggested that the xylem contribution could be much more important than commonly found with current methods. The small contribution of xylem to tomato fruit growth is explained by its high hydraulic resistance in the pedicel, which depends on the number and diameter of vessels (Nobel, 1983). Several studies have questioned the continuity of hydraulic resistance in the tomato fruit pedicel, which would contribute to the major hydraulic resistance of the pathway to the fruit (Mazzeo, 2008). van Ieperen et al. (2003) showed that the xylem resistance tends to increase throughout tomato fruit development in particular at the fruit abscission zone (AZ) of the pedicel, which contains few differentiated vessels. Indeed, the xylem cross-sectional area is significantly reduced at the AZ, relatively to the rest of the pedicel (Lee, 1989). In agreement, an estimation of the axial hydraulic conductance of the pedicel by the Hagen-Poiseuille law on the basis of anatomical observations of the area and diameter of xylem vessels suggests that the resistance increases at least two orders of magnitude along the pedicel (Rancic et al., 2010), due to narrower xylem vessel elements on the fruit side (Rancic et al., 2010).

In conditions of water deficit, the high hydraulic resistance in the pathway between stem and fruit would prevent fruit shrinkage (Malone and Andrews, 2001), though xylem backflow from the fruit were reported during the hottest hours of the day (Guichard et al., 2001). Stain diffusion experiment showed that a large proportion of xylem vessels are not functional and that water deficit affects the number and size of the elements of the functional xylem in the fruit pedicel, especially in the zone near the fruit (Rancic et al., 2008). Malone and Andrews (2001) confirmed the high hydraulic resistance between stem and fruit, but showed that xylem is functional and continuous throughout the tomato pedicel and estimated that more than $90 \%$ of the hydraulic resistance would occur within the fruit pericarp. 
On their whole, these studies point out the need for a better assessment of the area and conductivity of conducting tissues and their variabilities, in order to understand water fluxes between stem and fruit. In this perspective, process-based models could help integrating knowledge to analyze the effects of environmental and genetic factors on fruit growth. A modeling approach of water flow through the pedicel and within the fruit suggested that water importation in the young growing tomato could be limited mainly by the pedicel resistance and the phloem conductivity, linked to sap viscosity (Bussières 2002 ; Bussières et al., 2011). In agreement, a study on the conception of tomato fruit ideotypes adapted to water deficit based on the Virtual Fruit model (Fishman and Génard 1998 ; Liu et al 2007) led to the hypothesis that the conductance of the conductive tissues in the pedicel could be an interesting adaptation trait (Constantinescu et al., 2016). The Virtual Fruit model is a biophysical model of fruit expansion, which has been further extended to describe the longitudinal water fluxes in the pedicel and both longitudinal and radial water fluxes in the fruit (Hall et al. 2013). Yet in this model, the xylem and phloem conductances are parameterized in a rather empirical way, assuming proportionality between the exchange surfaces of xylem and phloem in the fruit and the fruit surface, and constant xylem and phloem conductivities in the pedicel. Experimental data are needed to evaluate and validate such hypotheses. More generally, conducting tissue area, conductivity and viscosity interact, but none of these variables is easily measured, and they are usually predicted empirically in growth models, while they become overriding to simulated fluxes in stress conditions. Combining anatomical observations and flow measurements may help to address the issue of resource transport in the plant. To this aim, MRI, a direct non-destructive method has been successfully applied to study water fluxes in the plant (Van As et al., 2007), However, very few information is available on the genetic variability of conducting tissues and on the impact of environmental factors. For instance, in tomato, high light decreases the xylem contribution to fruit growth (Hanssens et al., 2015) and water deficit impacts the size of xylem vessel (Rancic et al., 2008). But the extent to which this contributes to the variations in fruit fresh and dry masses is unclear.

In the present study we combined histological observations, modeling and MRI measurements in order to understand how the structural and functional properties of conducting tissues may affect fruit growth. An image analysis pipeline was developed to measure xylem and phloem cross-sectional areas and to better characterize anatomical organization in tomato pedicel and main stem. The variability of the conductive tissue areas in the pedicel was measured on contrasting genotypes and in response to water deficit. Then, this variability was implemented in the Virtual Fruit model, in order to evaluate its contribution to variations in fruit fresh and dry masses. In parallel, MRI measurements of water fluxes in the main stem were used to assess the proportion, the diameter and the theoretical conductance of active vessels.

\section{2 - MATERIAL AND METHODS}

\section{1 - Plant material}

The study investigated 11 contrasted genotypes of tomato (Solanum lycopersicum L.): West Virginia 106 (WVA106), a cherry tomato cultivar, the 8 parent genotypes of the MAGIC TOM population (Cervil, Criollo, Ferum, LA0147, LA1420, Levovil, PlovdivXXIVa (here called Plovdiv), Stupicke Polni Rane (here called Stupicke)) (Pascual et al., 2015 ; Ripoll et al., 2016), the wild-type of the Heinz genotype and the «Jointless » of Heinz with no abscission (Mao et al., 2000). All plants were grown in a heated glasshouse (18 $+/-2{ }^{\circ} \mathrm{C}$ night $/ 22+/-4{ }^{\circ} \mathrm{C}$ day) in Avignon, in $3 \mathrm{~L}$ pots filled with humus (Klasmann, Substrat SP $15 \%$ ) and automatically watered with a commercial nutritive solution (Liquoplant Rose, Plantin, Courthézon, France). Side-shoots were removed and trusses were pollinated with a vibrator three times a week.

Except for the water deficit (WD) experiment, all plants were irrigated in order to match the evaporative demand (control condition). To assess the impact of WD on the conductive tissue, WVA106 plants were grown under three scenarios of irrigation: control, $-50 \%$ of control (light WD) and $-65 \%$ of control (severe $\mathrm{WD}$ ). In case of severe $\mathrm{WD}$, the reduction was applied either 15 days before the truss anthesis (early severe WD) or 4 days after the truss anthesis (late severe WD). In case of light WD, the reduction was applied 15 days before truss anthesis (early light WD).

All measurements were carried out on reproductive plants bearing 7 to 10 fruiting trusses. 


\section{2 - Pedicel and fruit growth}

The pedicel and the fruit diameters of the second proximal (close to the stem) fruits of trusses that developed concomitantly on all genotypes were measured with a caliper throughout development (from fruit set to maturity). The pedicel diameter was measured at three positions: at the abscission zone (AZ) level, before $\mathrm{AZ}$ on the stem side $(\mathrm{AZs})$, and after $\mathrm{AZ}$ on the fruit side (AZf). At the fruit ripening stage, pedicels were sampled and immediately fixed and processed as described below for histological analysis (number of repetitions are indicated in figure legends). The fruits were weighted and dried in a ventilated oven to determine their dry mass and water content.

\section{3 - Histological pipeline to quantify the conductive tissue areas}

A histological pipeline was developed to characterize the conductive elements and study the pedicel and stem anatomy. In a first step, the fresh sample was fixed in Clarke's fixative solution (9 volumes of absolute ethanol in 1 volume of acetic acid) to keep the tissue structure (Sharma, 1956). Then, samples were embedded in a 5\% agarose gel (Ref. LE-8200-B Sigma) and transversally cut using a vibratome (Microm HM 650 V) (thickness of $60 \mu \mathrm{m}$ for the pedicel and $100 \mu \mathrm{m}$ for the main stem). Slices were treated with a bleach solution (at $36^{\circ}$ chlorogenic) to remove the cell content and increase the contrast among tissues. Histological sections were stained overnight with a FASGA solution dilu-ted at $1 / 7$ (Tolivia and Tolivia, 1987), then rinsed in a $50 \%$ glycerol solution. FASGA mother solution was composed of $2 \mathrm{~mL}$ of Safranine solution at $1 \%, 14 \mathrm{~mL}$ of a Alcian Blue solution at $0.5 \%, 1 \mathrm{~mL}$ of acetic acid, $30 \mathrm{~mL}$ of glycerin and $19.5 \mathrm{~mL}$ of distilled water.

Glass slides were converted into high-resolution digital data with a Nanozoomer RS slide scanner (NanoZoomer Digital Pathology System, Hamamatsu, Japan).

A plugin (PHIV_Tomate_toolset) was developed under Fiji (Shindelin et al., 2012) to calculate the number and the area of xylem and phloem vessels. Pedicel transversal sections were divided in five anatomical zones corresponding to (from the outer surface to the center): cortex, external phloem, secondary xylem, internal phloem and pith (Fig. 1). The masks of the tissues were achieved semi-automatically with the plugin and then corrected manually (if necessary) before calculating the areas of each tissue.

\section{4 - MRI measurements of in vivo fluxes and area of functional xylem vessels}

The area of active xylem vessels in the main stem was measured by Magnetic Resonance Imaging (MRI) and the water flux was directly assessed. The method relies on Nuclear Magnetic Resonance (NMR) principles, based on the electromagnetic properties of the spin of nuclei. In the present study, experiments were performed on a Agilent MRI scanner working at $9.4 \mathrm{~T}$ corresponding to $400 \mathrm{MHz}$ for the ${ }^{1} \mathrm{H}$ nuclei, the most commonly used atom in MRI experiments, which gives direct information on the tissue water content and its dynamic (Van As, 2007). The experimental set-up on living systems is based on a horizontal MRI scanner forcing the plant to lie horizontally in a cradle, moving inside a $10 \mathrm{~cm}$ diameter clear bore magnet. The MRI scanner is located in an air-conditioned room $\left(20^{\circ} \mathrm{C}\right)$, and a heater regulates the temperature at $25^{\circ} \mathrm{C}$ in the center of the magnetic field. WVA106 plants were transferred from the glasshouse to an indoor growth chamber at least one week before MRI measurements in order to adapt to a $12 \mathrm{~h}$-daylight period (PPFD $300 \mu \mathrm{mol} \mathrm{m}{ }^{-2} \mathrm{~s}^{-1}$ ) and $22^{\circ} \mathrm{C}$ temperature conditions.

For MRI experiments, an openable saddle-coil designed for measuring samples up to $14 \mathrm{~mm}$ diameter was installed around the tomato stem. The stem part that was in the coil was sampled after MRI measurements and processed through the histological pipeline.

A flow-MRI method involving the slice selectivity in the context of a multi spin echo sequence called flip-flop (Buy et al., 2018) was applied to measure water fluxes, both in upward and downward directions. Two pulse sequences measuring inflow and outflow weighted images of the same slice were performed, allowing slow flow sensitive imaging. With this method, the xylem fluxes could be mea-sured in the main stem of the plant as well as the active areas. The mean flux velocity was assessed by fitting the experimental data with the following expression : 
$F F_{\text {signal }}=r \cdot M_{0} \cdot \exp \left(-\frac{\mathrm{TR}}{T_{1}}\right)(1)$

Where $\mathrm{M}_{0}$ represents the total magnetization, $\mathrm{T} 1$ the longitudinal relaxation time $(\mathrm{s})$ and $\mathrm{r}$ the inflow factor which depends on sap flow hypothesis (Buy et al., 2018). Using a Poiseuille model, it can be shown that for a laminar flow, $\mathrm{r}$ is proportional to the mean velocity $v_{\text {mean }}$, the repetition time TR (s) and the inverse of the slice thickness. Matlab routines were developed to process MRI measurements at different TR values providing a map of flux velocities and of the active surfaces of the conductive xylem vessels that was compared with histological data. A particular attention was paid to registered inflow and outflow images before computing the various maps.

\section{5 - Virtual Fruit model}

The Virtual Fruit was used to integrate histological and MRI data and assess the potential impact of conducting tissues on fruit growth and composition. This process-based model simulates the water and dry matter accumulation during fruit growth, considering the fruit as one big cell connected to the stem by the pedicel conducting tissues (Fishman and Génard 1998 ; Liu et al., 2007 ; Hall et al., 2013). The model inputs are two climatic variables: air temperature and humidity and two plant variables: stem water potential and phloem sap concentration in sugars. Water and sucrose move from the stem to the fruit through the pedicel vasculature, and then within the fruit through composite membranes. Changes in fruit water content over time are calculated as the sum of xylem and phloem net influx, minus the water outflow due to fruit transpiration.

In the pedicel, the phloem flow depends on the pedicel phloem conductance (Lp1) and on the turgor pressure and osmotic potential difference between the stem and the fruit (Hall et al., 2013). The xylem flow is driven by the hydrostatic pressure difference between the stem xylem and the fruit xylem and depends on the pedicel xylem conductance (Lx1). In previous studies, Lp1 and Lx1 were assumed to remain constant during pedicel and fruit growth. In the present study, they were re-estimated based on the measured phloem and xylem areas.

In the fruit, the xylem and phloem fluxes are calculated from non-equilibrium thermodynamic equations involving the surface of exchange, which is assumed to be proportional to the fruit surface area. It also involves hydraulic conductivity, solute reflection coefficients of the membrane separating the fruit from the conducting tissues of the pedicel and hydrostatic and osmotic pressures, calculated from the molar concentrations of osmotically active compounds (Liu et al., 2007).

The Virtual Fruit model was calibrated for the genotype WVA106 based on independent data of fruit dry and fresh masses collected on control plants grown in a glasshouse. The NSGA-II algorithm (Deb et al., 2002) was used to find the best combination of parameters which predicts the experimental dry and fresh masses. The outputs of this algorithm depend on the random initial values chosen, so the optimization was repeated 10 times.

\section{6 - Statistical analysis}

Kinetic and histological data were analyzed with the $\mathrm{R}$ software (R Core Team, 2017).

The pedicel diameter kinetics were fitted using a segmented model (Muggeo, 2008). The fruit diameter kinetics were fitted using a Gompertz model with the following equation:

$f(x)=a \cdot \exp \left(-\exp \left(-\frac{x-c}{b}\right)\right)(2)$

Kruskal-Wallis tests were used to compare the relative area of the conductive tissues, the pedicel diameter, the fruit fresh mass and dry matter content, between control and the different WD treatments. The flow-MRI data processing and analysis were performed under Matlab programming language (MATLAB, 2016).

\section{3 - RESULTS}

3.1 - Histological quantification of the conducting tissues in tomato pedicel 
The histological pipeline described above was first applied on two genotypes, Heinz wild-type and Heinz Jointless, that differed by the presence (wild-type) or absence (Jointless) of an abscission zone (AZ). The increase in pedicel diameter over fruit development was not significantly different before and after the AZ, and between the wild-type and the Jointless (data not shown). The final fruit diameter, pedicel diameter and dry matter content (DMC) of the mutant were equal to $44.2+/-6.2 \mathrm{~mm}, 3.8+/-0.6 \mathrm{~mm}$ and $7.9+/-$ $0.5 \%$, respectively. For the wild-type, they were equal to $49.9+/-2.8 \mathrm{~mm}, 4.1+/-0.3 \mathrm{~mm}$ and $6.6+/-0.4$ $\%$, respectively.

The histological properties were measured on the stem side of the pedicel (AZs) and on the fruit side of the pedicel (AZf) (Fig. 2). At the AZ level, the xylem tissues were clearly non-lignified in the wild-type and the area of each tissue could not be quantified (Fig. 2A). On the contrary for the mutant a continuous lignification of the secondary xylem was observed at the level of the theoretical AZ (defined by the pedicel curvature) (Fig. 2B). Comparing AZf and AZs, the proportion of secondary xylem to the pedicel section was lower on the fruit side than on the stem side whereas the proportion of cortex was higher. This was observed both in the wild type (Fig. 2C) and the mutant (Fig. 2D), but differences were significant for the wild type only $(\mathrm{P}=0.013)$. Interestingly in the mutant, the proportions of tissues in the theoretical $\mathrm{AZ}$ and in the AZs were very similar and the proportions of conductive tissues changed along the pedicel, independently of the presence or absence of AZ.

Between the two genotypes, the proportions of tissues were quite comparable (Fig. 2C and D). On average, the proportions of cortex, secondary xylem, pith, external phloem and internal phloem on the fruit size were about $48.6 \%, 20 \%, 15.6 \%, 9.1 \%$ and $6.8 \%$, respectively. On the stem side, these ave-rage proportions of tissues were about $37.2 \%, 29.7 \%, 15 \%, 11 \%$ and $7.1 \%$, respectively.

In the following, the genotypic and environmental variability of tissue proportions were investigated on the AZf for the sake of simplicity.

\section{2 - Assessment of the genotypic variability of pedicel diameter and conductive tissue areas}

\subsection{1 - Pedicel and fruit growth kinetics}

The pedicel growth and anatomy were investigated in relation to fruit expansion on the two Heinz genotypes and on the eight MAGIC TOM parents. Whatever the genotype, the pedicel stopped growing as the fruit reached about $29 \%$ of its age at maturity (Fig. 3A). In contrast, fruit diameter kinetics were genotypedependent (Fig. 3B). In particular, the age at which the fruit growth rate was maximum ranged from $19 \%$ to $37 \%$ of the age at maturity, that is 10 to 27 days after anthesis (daa), the earliest being Heinz wild-type, Heinz Jointless and Cervil and the latest being LA1420.

Interestingly, depending on genotypes the increase in pedicel diameter stopped before or after the fruit expansion rate was maximum, suggesting that the conductive tissue area was set synchronously or not with the fruit demand.

\subsection{2 - Final fruit mass and pedicel conducting tissue areas}

To further investigate the genotypic variability of the pedicel conducting tissues at maturity, a histological analysis was performed on 11 genotypes (the previous 10 genotypes, adding WVA106). Genotypes were divided in three classes of fresh mass (FM) (Fig.4A): small fruit (FM > 15g) composed by Cervil, WVA106 and Criollo, large fruits (FM >100g) composed by Ferum, LA0147 and Levovil, and medium fruits in between, including LA1420, Plovdiv, Heinz Jointless, Stupicke and Heinz wild-type. The fruit DMC ranged from 5.4 to $13.2 \%$. A negative correlation was observed between the fruit FM and the DMC with strong variations within each class, for instance between Criollo and Cervil or between Ferum and Levovil (Fig. 4B). The final pedicel diameter varied by a factor two (Fig. 4C). Large fruits had on average a higher pedicel diameter than small and medium fruit size genotypes, although Cervil, Heinz Jointless and wild-type had a larger pedicel than other genotypes from the same class.

The area of the different conducting tissues, expressed in absolute values, varied a lot among genotypes (Fig. 
$4 \mathrm{D})$. Among the three conductive tissues, the secondary xylem area was always the most important and the internal phloem area was the smallest. The mean absolute area of secondary xylem varied by a factor of 4.3 between WVA106 (lowest value) and Heinz Jointless (highest value), the mean area of external phloem varied by a factor of 4.8 between LA1420 and Heinz wild-type and the area of internal phloem varied by a factor of 6.2 between Criollo and Heinz wild-type. These variations were reduced within each fruit class, and the medium fruit group was the most variable (factor of 4), due to the Heinz genotypes.

When expressed in relative proportions of the pedicel section, the cortex was always the most important tissue, followed by the secondary xylem or by pith depending on genotype, whereas the external and internal phloem represented the lowest proportions (Fig. 4E). Focusing on the conductive tissues, the proportion of secondary xylem varied from $15 \%$ (Cervil) to $31 \%$ (Heinz Jointless), the proportion of external phloem varied from $6 \%$ (Cervil) to $11 \%$ (Stupicke) and the proportion of internal phloem varied from 2\% (Cervil) to $7 \%$ (LA0147).

To better explore the links among variables, a principal component analysis (PCA) was performed on the proportions of tissues, the pedicel diameter, the fruit FM and DMC (Fig. 5). The first axis (43\% explained variance) separates individuals with high fruit $\mathrm{FM}$, high pith and internal phloem proportions and high pedicel diameter $(\mathrm{PCA} 1>0)$, from individuals with high proportions of cortex and high DMC $(\mathrm{PCA} 1<0)$. The second axis (23\% explained variance) mostly represents individuals with high proportions of secondary xylem and external phloem $(\mathrm{PCA} 2<0)$. The projection of the 11 genotypes on the first plane evidenced four groups of genotypes. A group located on the right upper corner was composed of large fruit genotype (Levovil and LA0147) and one medium fruit genotype (Heinz wild-type) with relatively low DMC ( $<7 \%)$. This group was associated with a low cortex proportion, high pedicel diameter and high pith and internal phloem proportions. On the opposite, Cervil the genotype with the smallest FM and highest DMC was positioned in the left upper corner. It was associated with a high cortex proportion, a small pith and internal phloem proportion, a small pedicel diameter, and small proportion of external phloem and secondary xylem. The other genotypes of the small fruits group are not well represented on this PCA. Two other atypical genotypes discriminated on the second axis. Ferum, a genotype from the large fruits group with a relatively high DMC is associated with a low proportion of external phloem and secondary xylem, in contrast with LA1420, a genotype with a relatively low DMC compare to its small size $(\mathrm{FM}<25 \mathrm{~g})$.

The third axes and the fourth explained $10 \%$ of the variance each, but did not discriminate well the genotypes.

3.3 - Water deficit impact on the conductive tissues of the tomato pedicel in relation to fruit growth

Water deficit (WD) is known to affect the fruit fresh mass and dry matter content of tomato, but little is known about the effect on the conductive tissues. The effect of three scenarios of WD on the fruit growth and pedicel anatomy were investigated on one genotype (WVA106). All three scenarios signi-ficantly reduced the fruit FM $(-12.9 \%,-22.6 \%$ and $-26.9 \%$, for the early light, early severe and late severe WD, respectively) and significantly increased the DMC $(+24.3 \%,+24.3 \%$ and $+16.5 \%$, for the early light, early severe and late severe WD, respectively) (Fig. 6A, B). The pedicel diameter was significantly lower in the early severe WD than in the control treatment $(-21.1 \%)$ and was not significantly affected in the two other scenarios (Fig. $6 \mathrm{C})$.

The absolute and relative areas of the different tissues were significantly affected by the early WDs only (Fig. 6D, E). The early light WD provoked a significant reduction of the pith surface compared to the control (-53\%). The early severe WD provoked a significant reduction of the secondary xylem (-54\%), the pith $(-63 \%)$, the external phloem $(-55 \%)$ and the internal phloem $(-51 \%)$ areas. In proportions of tissues in the pedicel section, the early light WD significantly increased the proportions of cortex $(+12.8 \%)$ and decreased the proportions of pith (-39.4\%) and internal phloem (-16.6\%). The early severe WD significantly decreased the proportions of secondary xylem $(-18.6 \%)$ and pith $(-33.3 \%)$, and significantly increased the cortex proportion $(+21.3 \%)$, whereas the phloem tissues were not affected. In this treatment, the strong reduction of the pedicel diameter could explain the significant effect on the absolute areas (Fig. 6C).

3.4 - Model-based analysis of the contribution of conducting tissues to the genotypic variability and water 
deficit impact on tomato fruit mass

The Virtual fruit model was calibrated for WVA106 in well-watered conditions based on fresh and dry mass measurements (Fig. 7). Nine parameters involved in the control of FM and dry mass (DM) were estimated, in which two were involved in the cellular expansion, two in the active sugar uptake, one in the fruit sink strength and four in the fruit and pedicel conductivity. The simulation covers a 28-day period of rapid fruit growth after cell division (starting at 10 days after anthesis).

\section{4 .1 - Simulation of the genotypic variability}

The phloem and the xylem conductances (Lp1 and Lx1) were assumed to be proportional to the xylem and phloem areas measured in this study. Since there is no distinction between internal and external phloem in the model, the internal and external phloem areas were simply added. To account for the genotypic variability, we reported on Lx1 and Lp1, the maximum variations of xylem and phloem areas observed between WVA106 and the other genotypes (Fig. 4D): the secondary xylem area varied from 0 to $+332 \%$ and the phloem area varied from $-6 \%$ to $+347 \%$. The final fruit FM and DMC simulated for WVA106 were 9.86g and $8.3 \%$, respectively (Fig. 7A). A 10\% decrease of Lx1 and Lp1 did not strongly affect these values (9.54 g and 8.4\%, respectively). On the contrary, a 350\% increase of Lx1 and Lp1, increased the simulated FM (15.24 g) and decreased the DMC (6.2\%). Thus, according to the Virtual Fruit model, the genotypic variability in Lx1 and Lp1 deduced from the observed variability in conducting tissue areas, contributed very modestly to the actual genotypic variations in fruit FM and DM (Fig. 4D).

\subsection{2 - Simulation of the water deficit scenarios}

The same approach was applied for the WD experiment (WVA106 genotype). For the early light WD, Lx1 and Lp1 were reduced by $23 \%$ and $36 \%$, respectively according to the reduction of xylem and phloem areas (Fig. 6D). For the early severe WD, both Lx1 and Lp1 were decreased by 54\%. For the late severe WD, Lx1 and Lp1 were respectively decreased by $28 \%$ and $27 \%$. All other parameters were unchanged and kept identical for both treatments. The simulated FM, DM and DMC in response to these WD deficit scenarios are represented on Fig. 7B.

The simulated FM at maturity was $9.86 \mathrm{~g}$ in the control scenario (experimental data: $9.3+/-1 \mathrm{~g}$ ), $8.84 \mathrm{~g}$ in the early light WD scenario (experimental data: $8.1+/-0.4 \mathrm{~g}), 7.67 \mathrm{~g}$ in the early severe WD scenario (experimental data: 7.2 +/- $0.5 \mathrm{~g}$ ) and $8.91 \mathrm{~g}$ in the late severe WD scenario (experimental data: $6.8+/-$ $0.6 \mathrm{~g})$. Thus, for the early light and early severe WD, changes in Lx1 and Lp1 led to a reduction of the simulated fruit FM of the same order than that actually measured. On the opposite under late severe WD the reduction of the simulated FM (-9.6\%) was about three times lower than that measured $(-26.8 \%)$.

The simulated final DMC was $8.3 \%$ in the control, $8.4 \%$ in the early light WD scenario, $8.9 \%$ in the early severe WD scenario and $8.6 \%$ in the late severe WD scenario. According to the Virtual Fruit model, the reduction of the xylem and phloem conductance at the pedicel level hardly impacted the simulated DMC for the three scenarios whereas an increase of $24 \%$ (early light WD), $23 \%$ (early severe WD) and 16\% (late severe WD) was experimentally measured (Fig. 6B).

3.5 - MRI quantification of the proportion of functional tissues and their conductance in the main stem

The percentage of functional tissues was assessed by MRI at the stem level, in order to benefit from a better resolution (Fig. 8). Under the MRI experimental conditions, the mean flux velocity measured in the tomato stem with the flip-flop method (Buy et al., 2018) was $0.469 \mathrm{~mm} \mathrm{~s}^{-1}$ and the active area of conducting tissues was $1.8 \mathrm{~mm}^{2}$ (Fig. 8C). The resulting water flow was equal to $0.844 \mathrm{~mm}^{3} \mathrm{~s}^{-1}$, which approximatively corresponds to $3.04 \mathrm{~g} \mathrm{~h}^{-1}$.

Using the inflow MRI image (Fig. 8A), it was possible to measure the diameter of active xylem vessels. 242 active vessels were counted with a mean diameter of $93.8 \mu \mathrm{m}$ (Fig. 8D), which represented a total area of $1.74 \mathrm{~mm}^{2}$. 
The comparison of the inflow MRI image (Fig. 8A) with the histological slice (Fig. 8B) indicated that the active conductive tissues were xylem vessels and that only part of them was active.

The area of secondary xylem vessels (the area of secondary xylem tissue without the fibers and parenchyma cells areas) on the histological section was estimated at $3.53 \mathrm{~mm}^{2}$, which is twice the active area evidenced by MRI. This means that around $50 \%$ of the xylem vessel area was active under the MRI experimental conditions.

The number of secondary xylem vessels (diameter higher than $10 \mu \mathrm{m}$ ) counted on the whole histological section was about 1222 and their mean diameter was $53.4 \mu \mathrm{m}$ (Fig. 8D). The comparison of the distribution of xylem vessel diameters of the whole stem section with the distribution of active xylem vessels indicates that the active vessels are mainly the largest ones. A theoretical xylem conductance was estimated from the vessel diameter using the Hagen-Poiseuille relation (Calkin et al., 1985):

$k_{h}=\left(\frac{\pi}{128 \eta}\right) \sum_{i-1}^{n}\left(d_{i}^{4}\right)(3)$

where $\eta$ is the dynamic viscosity of the fluid in MPa $\mathrm{s}, \mathrm{d}$ the diameter in $\mathrm{m}$ of the ith vessels and $\mathrm{n}$ the number of vessels. We considered here that the viscosity of xylem sap was equal to that of water, so $\eta=1$ $10^{-9} \mathrm{MPa}$ s at $20^{\circ} \mathrm{C}$. Using the diameter of the active vessels, the estimated theoretical conductance of active vessels was $5.610^{-7} \mathrm{~m}^{4} \mathrm{MPa}^{-1} \mathrm{~s}^{-1}$ and the theoretical conductance of the xylem vessels of the whole stem cross section was $8.010^{-7} \mathrm{~m}^{4} \mathrm{MPa}^{-1} \mathrm{~s}^{-1} .97 \%$ of the theoretical conductance of the xylem vessels of the whole stem was due to vessels that have a diameter higher than $50 \mu \mathrm{m}$, even though more than $50 \%$ of the vessels have a diameter below $50 \mu \mathrm{m}$.

\section{4 - DISCUSSION}

Tomato fruits are important sink organs whose mass varies during development by one or two orders of magnitude depending on genotypes and according to the water and carbohydrate importation by the fruit. The fruits are supplied by a complex system of xylem and phloem tissues, which align along the pedicel and ramify within the fruit tissues (Belda and Ho, 1993). Although the genetic variability in fruit fresh and dry masses has been widely studied (e.g. Bertin et al., 2009), the variability in the transport pathway has been rarely described. Similarly the effect of water deficit on fruit mass and pedicel anatomy are reported (Rancic et al., 2008), but the actual contribution of the conductive tissues was hardly assessed quantitatively. In the present study, anatomical, histological, modeling and MRI methods were combined to assess the kinetics of pedicel and fruit growth, to evaluate the contribution of the pedicel tissues to the variations of fruit mass and dry matter content in response to water deficit and genotype and to give new insight at the vessel level. The study confirms the capacity of flow-MRI to assess sap fluxes in planta (Van As, 2007 ; Van As et al., 2009 ; Buy et al., 2018) and highlights the interest to combine such method with histological and modeling approaches to better understand hydraulic regulations in the plant architecture. It also provides an original overwiew of the genotypic variations of pedicel anatomy on a collection of contrasted genotypes whose fresh mass varied from a few grams to more than $100 \mathrm{~g}$ and dry matter content ranged from 5.4 to $13.2 \%$.

4.1 -The decrease in the conducting tissues area along the pedicel rather than the discontinuity at the abscission point contributes to the reduction of conductivity along the transport pathway to the fruit

Many studies focused on the hydraulic resistance of the tomato pedicel and its impact on the supply of water and minerals, calcium in particular, to the fruit (Belda and Ho, 1993 ; Malone and Andrews, 2001 ; van Ieperen et al., 2003 ; Rancic et al., 2010). In the present study, a clear discontinuity of the xylem lignification was observed at the abscission point, in agreement with a previous study on this genotype (Szymkowiak and Irish, 1999), together with a decrease of the xylem proportion along the pedicel which was independent of the presence or absence of AZ. Therefore, both the xylem discontinuity and the decrease of secondary xylem area along the pedicel may contribute to increase the hydraulic resistance. Despite the absence of AZ, the Jointless mutant had smaller fruit FM and a higher DMC, which is counter-intuitive since the hydraulic resistance along the pedicel should be lower for the mutant than for the wild-type (van Ieperen el al., 2003). 
The mutant also had a smaller pedicel diameter compared to the wild-type with similar proportion of tissues. So the lower areas of xylem and phloem tissues likely explained the lower fruit FM of the mutant. These results support the hypothesis that the non-lignification of tissues in the AZ does not necessarily reduce the hydraulic conductivity and that only the mechanical components of the xylem (fibres and xylem parenchyma) may be interrupted (Malone and Andrews, 2001). They also suggest that the increasing hydraulic resistance along the pedicel partly results from a decreasing area of conductive tissues in agreement with Lee (1989) and Rancic et al. (2010).

In the future, a better assessment of the functionality and size of active vessels will allow to measure the gradient of conductivity along the pedicel. Flow-MRI appeared as a promising tool, but up to now the quantitative method applied in this study (Buy et al., 2018) is not sensitive enough for measuring low fluxes in small vessels. Windt et al. (2009) used diffusion weighted MRI to quantify the xylem and phloem contributions to fruit growth and suggested that the xylem contribution would be much more important than commonly found with indirect methods. However in this study, measurements were not done at the pedicel level, but at the peduncle level, due to the complexity of adapting a coil that fits the pedicel. Thus improving the MRI sensitivity and adapting coils to small pedicels may allow to conclude on the main route of water transport to the fruit and to estimate in vivo a pedicel conductance. These measurements would improve the Virtual Fruit model formalisms, by allowing to decorrelate the pedicel and the fruit resistances.

\section{2 - The effect of water deficit on the pedicel anatomy and areas of conductive tissues partially explains its effects on fruit mass and dry matter content}

WD is known to decrease the fresh mass of tomato and to increase the dry matter content mainly due to an increase in the phloem sap concentration and to a decrease in the water flow to the fruit (Ho et al., 1987 ; Guichard et al., 2001), depending on stress intensity and duration (Ripoll et al., 2016). In the present study, the early WD treatments affected the whole period of fruit development including cell division, while the late WD treatment impacted only the cell expansion period. The reduction of FM related to stress intensity rather than to the period of application, suggested that the cell expansion processes were the most affected in agreement with several studies (Mahdid et al., 2011; Ripoll et al., 2016; Koch et al., 2019). On the contrary the increase in DMC was more sensitive to the period of WD occurrence than to the intensity (Fig. 6B). These results could be partially explained by the growth and histology of the pedicel. In case of early WD, the pedicel diameter was reduced under severe stress and the proportions of tissues were affected at both intensities with more cortex and less pith, but also less internal phloem and secondary xylem for the light and severe water deficits, respectively. The similar fruit DMC at both intensities suggests that xylem and phloem fluxes were affected in similar proportions or that the transpiration rate was reduced in proportion to the xylem influx (Guichard et al., 2001). The model-based analysis suggested that the observed reduction in xylem and phloem tissues alone could account for most of the reduction of fruit FM observed under early WD, but not for the increase in DMC. This suggests that an efflux of water, either by transpiration or back-flow to the stem may have occurred as suggested in previous studies (Guichard et al., 2005). Some driving variables such as stem water potential, sap sugars concentration could also be greatly affected by the water deficit, but were not considered here. Furthermore, variation in the sap viscosity could also be involved (Bussières 2002 ; Constantinescu et al., 2016) and could greatly affect the simulated fresh and dry masses.

In case of late WD, the pedicel was less affected since its development was probably already achieved when stress occurred, as suggested by Fig. 3. In that case the reduction of FM and increase in DMC could not be attributed to the effect of WD on the pedicel conductivity, but rather to regulations of resource distributions at the plant level or to xylem back-flow of water from the fruit to the stem as observed in many studies and many species (Lang and Thorpe, 1989; Huguet et al., 1997 ; Guichard et al., 2005 ; Keller et al., 2006 ; Carlomagno, 2018 ; Constantinescu 2019). In line with this hypothesis, the model-based analysis of the late WD effects based on the reduction of pedicel conductances failed to predict the observed fruit FM.

The present study confirmed that several factors are involved in the fruit response to WD and that complex interactions occur depending on stress intensity and timing. The modeling integrative approach is relevant 
to address this complexity, but it also requires adequate phenotyping tools to measure the areas of active vessels in the plant architecture. Indeed the absolute surfaces of tissue differ from the active surface. In the main stem, only $50 \%$ of the vessel area was found to be active under the MRI experimental conditions. Furthermore, rather than the total conductive area, the number of conducting elements and their diameter distribution are decisive for the conductance. From the present observations, the diameter of vessels was quite variable at the stem level with four main areas of large active vessels separated by small xylem vessels (Fig. 8). Several studies showed that WD decreased the diameter of xylem vessels, in tomato pedicel (Rancic et al., 2008), in grape (Lovisolo and Schubert, 1998) and in maize (Abdel-Rahim et al., 1998 ; Liu et al., 2019). On poplar tree, Arend and Fromm (2007) showed that WD provoked a decrease in vessel size, but also an increase in the number of newly formed vessel cells. In boreal trees, Schreiber et al (2015) showed that vessels diameters were highly plastic in response to the environment and climatic conditions. Such variations may greatly affect the tissue conductance, as shown here at the tomato stem level.

The estimated theoretical conductance at the main stem level was about 10 times that estimated at the pedicel level by Rancic et al. (2010), which makes sense since the main stem diameter is at least 10 times higher than the pedicel diameter. The present MRI observations indicate that active vessels are the largest ones (Fig. 8). Even though a large number of tiny vessels are visible over the whole stem section, no flux was measured by MRI in these vessels. It is not possible to conclude whether they were not active or whether the MRI resolution was insufficient, but we showed that the contribution of these vessels to the theoretical conductance is very low. This suggests that investigating only the largest vessels may be sufficient to estimate the tissue conductance. Another information brought out by the flow-MRI measurement is that only $60 \%$ of large vessels are active in the bottom of the main stem, in well-watered conditions. In the future this method will be applied to analyze the effect of WD on the number and diameter of active vessels, at different stem levels and possibly along the fruit pedicel.

\section{3 - Pedicel anatomical traits are worth being phenotyped for screening genetic resources}

Constantinescu et al. (2016) suggested that pedicel conductivity and active sugar uptake could be interesting traits to maintain FM of large-fruit genotypes under WD and increase their DMC under well-irrigated conditions. In the present study, a large genetic variability in the absolute surface of xylem and phloem was observed, which has never been reported before to our knowledge. The larger the areas, the larger the fruit mass, although some variation around this trend was observed within each class of FM. The variations in xylem and phloem areas alone could not account for the 100-fold difference in fresh mass between cherry tomatoes and large fruits, but the simulations showed that it could partly contribute. Indeed, simulations of fresh and dry masses and DMC were sensitive to phloem and xylem conductance in the pedicel, although other important parameters such as the initial fruit mass (Constantinescu et al., 2016) were not considered here. In proportion, the relative area of tissues varied by a factor 2 to 3 among genotypes, the internal phloem being the most variable tissue. The PCA suggested that large proportions of internal phloem are associated to large FM. At the tomato stem level, the upward movement of assimilates occurs mainly in the internal phloem and the downward movement in the external phloem (Bonnemain, 1966). Moreover fruit trusses are mainly supplied by the two leaves below the truss and the leaf above it (Bonnemain, 1966). These results are in line with the positive correlation observed between internal phloem proportion and fruit mass. On the contrary no clear relation was observed between the proportion of secondary xylem, neither with the fruit FM nor the DMC. Interestingly, the external phloem was not correlated to the internal phloem, but to the secondary xylem, and they discriminated the medium size genotypes.

The asynchronous development of fruit and pedicel is another issue. While the pedicel grew for a constant period of fruit development, which was similar for all genotypes, the kinetics of fruit growth was genotypedependent. For some genotypes, the pipework grows together with the sink and in other cases it is fixed before the demand reaches its maximum. Therefore, depending on genotype, the pedicel conductivity may be constant or variable during the period of rapid fruit expansion. More investigations are necessarily to confirm these data at the tissue and vessel levels and assess the consequences in terms of flow limitations.

\section{4 - Concluding remarks and perspectives}


On their whole, results suggested that the variations in structural and functional properties of conducting vessels are linked to the genotypic and environmental variations of tomato fruit fresh mass and dry matter content and highlight the importance of combining multiple methods to integrate information and to improve plant simulation models. In order to measure crucial factors such as the proportion of active vessels and variations in individual vessel diameter, flow-MRI was shown to be a promising tool, being an easy nondestructive method to phenotype the functional properties of conducting tissues.

\section{AUTHOR CONTRIBUTION}

M. C., B. B., E. A., C. B. and J. S. carried out experiments. C. G.-B., N. B. and J. S. designed the project. M. C., M. L., G. V., J.-L. V. and J. S. contributed to data analysis. N. B., C. G.-B. and J. S. wrote the manuscript. M. C., G. V. and J.-L. V. proofread the manuscript.

\section{ACKNOWLEDGEMENTS}

This work was carried out thanks to the support of the «Investissements d'avenir» program (Labex Agro:ANR-10-LABX-0001-01), under the frame of I-SITE MUSE (ANR-16-IDEX-0006) and thanks to the support of the department AgroEnv INRAE. We thank Guillaume Garcia and Thibault Crouzet for their contributions.

\section{References}

Abd El-Rahim M. F., Fahmy G. M., \& Fahmy Z. M. (1998). Alterations in transpiration and stem vascular tissues of two maize cultivars under conditions of water stress and late wilt disease. Plant pathology, 47(2), 216-223.

Arend M. \& Fromm J. (2007). Seasonal change in the drought response of wood cell development in poplar. Tree physiology, 27(7), 985-992.

Bertin N., Causse M., Brunel B., Tricon D. \& Genard M. (2009). Identification of growth processes involved in QTLs for tomato fruit size and composition. Journal of Experimental Botany, 60(1), 237-248.

Belda R. M. \& Ho L. C. (1993). Salinity effects on the network of vascular bundles during tomato fruit development. Journal of horticultural science, 68(4), 557-564.

Bonnemain J. L. (1966). Sur les modalites de la distribution des assimilats chez la tomate et sur ses mecanismes. COMPTES RENDUS HEBDOMADAIRES DES SEANCES DE L ACADEMIE DES SCIENCES SERIE D, 262(10), 1106-+.

Bussieres P. (2002). Water import in the young tomato fruit limited by pedicel resistance and calyx transpiration. Functional Plant Biology, 29(5), 631-641.

Bussieres P., Bertin N., Morris C. E., Vigne C., Orlando P., Glaux C., Sevenier V., Floret H., Bernadac J. \& Korownikoff S. (2011). High external sucrose concentration inhibits the expansion of detached tomato fruits grown in a novel semi-open device. In Vitro Cellular \& Developmental Biology-Plant, 47(6), 743-751.

Buy S., Le Floch S., Tang N., Sidiboulenouar R., Zanca M., Canadas P., Nativel E., Cardoso M., Alibert E., Dupont G., Ambard D., Maurel C., Verdeil J.-L., Bertin N., Goze-Bac C. \& Coillot C. (2018). Flip-flop method: A new T1-weighted flow-MRI for plants studies. PLoS One,13(3).

Carlomagno A., Novello V., Ferrandino A., Genre A., Lovisolo C. \& Hunter J. J. (2018). Pre-harvest berry shrinkage in cv 'Shiraz'(Vitis vinifera L.): Understanding sap flow by means of tracing. Scientia horticulturae, 233, 394-406.

Constantinescu D. (2019, December). Analyse et amelioration d'un modele mecaniste de croissance et composition du fruit: Etude de la variabilite genetique des mecanismes de croissance du fruit, conception d'ideotypes et analyse des effets des transferts d'eau et de la concentration des solutes sur la croissance du fruit (Doctoral dissertation, $\mathrm{PhD}$ thesis. University of Avignon). 
Constantinescu D., Memmah M. M., Vercambre G., Genard M., Baldazzi V., Causse M., Albert E., Brunel B., Valsesia P. \& Bertin N. (2016). Model-assisted estimation of the genetic variability in physiological parameters related to tomato fruit growth under contrasted water conditions. Frontiers in plant science, 7 , 1841.

Deb K., Pratap A., Agarwal S. \& Meyarivan T. A. M. T. (2002). A fast and elitist multiobjective genetic algorithm: NSGA-II. EEE transactions on evolutionary computation, 6(2), 182-197.

Fishman S. \& Genard M. (1998) A biophysical model of fruit growth: simulation of seasonal and diurnal dynamics of mass. Plant, Cell and Environment, 21(8), 739-752.

Guichard S., Bertin N., Leonardi C. \& Gary C. (2001). Tomato fruit quality in relation to water and carbon fluxes.

Guichard S., Gary C., Leonardi C. \& Bertin N. (2005). Analysis of growth and water relations of tomato fruits in relation to air vapor pressure deficit and plant fruit load. Journal of Plant Growth Regulation, 24(3), 201.

Hall A. J., Minchin P. E. H., Clearwater M. J. \& Genard M. (2013). A biophysical model of kiwifruit (Actinidia deliciosa) berry development. Journal of Experimental Botany, 64(18), 5473-5483.

Hanssens J., De Swaef T. \& Steppe K. (2015). High light decreases xylem contribution to fruit growth in tomato. Plant, cell $\&$ environment, 38(3), 487-498.

Ho L. C. (1996). The mechanism of assimilate partitioning and carbohydrate compartmentation in fruit in relation to the quality and yield of tomato. Journal of Experimental Botany , 1239-1243.

Ho L. C., Grange R. I. \& Picken A. J. (1987). An analysis of the accumulation of water and dry matter in tomato fruit. Plant, Cell ES Environment, 10(2), 157-162.

Huguet J. G., Genard M., Laurent R., Besset J., Bussi C. \& Girard T. (1997) Xylemic, phloemic and transpiration flows to and from a peach, Acta Horticulturae, 465, 345-353.

Keller M., Zhang Y. U. N., Shrestha P. M., Biondi M. \& Bondada B. R. (2015) Sugar demand of ripening grape berries leads to recycling of surplus phloem water via the xylem, Plant, Cell and Environment,38(6), 1048-1059.

Koch G., Rolland G., Dauzat M., Bediee A., Baldazzi V., Bertin N., Guedon Y. \& Granier C. (2019). Leaf Production and Expansion: A Generalized Response to Drought Stresses from Cells to Whole Leaf Biomass-A Case Study in the Tomato Compound Leaf. Plants,8(10), 409.

Lang A. \& Thorpe M. R. (1989). Xylem, phloem and transpiration flows in a grape: application of a technique for measuring the volume of attached fruits to high resolution using Archimedes' principle. Journal of Experimental Botany, 40(10), 1069-1078.

Lee D. R. (1989). Vasculature of the abscission zone of tomato fruit: implications for transport. Canadian Journal of Botany, 67(6), 1898-1902.

Liu H. F., Genard M., Guichard S. \& Bertin N. (2007). Model-assisted analysis of tomato fruit growth in relation to carbon and water fluxes. Journal of Experimental Botany, 58(13), 3567-3580.

Liu J., Kang S., Davies W. J. \& Ding R. (2019). Elevated [CO2] alleviates the impacts of water deficit on xylem anatomy and hydraulic properties of maize stems. Plant, cell \& environment, 43.

Lough T. J. \& Lucas W. J. (2006). Integrative plant biology: role of phloem long-distance macromolecular trafficking. Annual Reviews of Plant Biology, 57, 203-232.

Lovisolo C. \& Schubert A. (1998). Effects of water stress on vessel size and xylem hydraulic conductivity in Vitis vinifera L. Journal of Experimental Botany, 49(321), 693-70 0. 
Mahdid M., Kameli A., Ehlert C. \& Simonneau T. (2011). Rapid changes in leaf elongation, ABA and water status during the recovery phase following application of water stress in two durum wheat varieties differing in drought tolerance. Plant Physiology and Biochemistry , 49(10), 1077-1083.

Malone M. \& Andrews J. (2001). The distribution of xylem hydraulic resistance in the fruiting truss of tomato. Plant, Cell \& Environment, 24(5), 565-570.

Mao L., Begum D., Chuang H. W., Budiman M. A., Szymkowiak E. J., Irish E. E. \& Wing R. A. (2000). JOINTLESS is a MADS-box gene controlling tomato flower abscission zone development. Nature, 406(6798), 910-913.

MATLAB (2016). Version 9.0.0 (R2016a) . Natick, Massachussetts: The MathWorks Inc.

Mazzeo M. (2008). Xylem transport efficiency and calcium accumulation in fruit of Actinidia deliciosa: implications for fruit quality (Doctoral dissertation, $\mathrm{PhD}$ thesis. University of Basilicata, Potenza).

Muggeo V. M. (2008). Segmented: an R package to fit regression models with broken-line relationships. $R$ news, 8(1), 20-25.

Nobel P.S. (1983) Biophysical plant physiology and ecology, 608pp. W.H. Freeman and Company, New York.

Pascual L., Desplat N., Huang B. E., Desgroux A., Bruguier L., Bouchet J. P., Le G. H., Chauchard B., Verschave P. \& Causse M. (2015). Potential of a tomato MAGIC population to decipher the genetic control of quantitative traits and detect causal variants in the resequencing era. Plant biotechnology journal, 13(4), 565-577.

Rančić D., Quarrie S. P. \& Pećinar I. (2010). Anatomy of tomato fruit and fruit pedicel during fruit development. Microscopy: Science, Technology, Applications and Education, 2, 851-861.

Rančić D., Quarrie S. P., Terzić M., Savić S. \& Stikić R. (2008). Comparison of light and fluorescence microscopy for xylem analysis in tomato pedicels during fruit development. Journal of microscopy,232(3), 618-622.

R Core Team (2017). R: A language and environment for statistical computing. R Foundation for Statistical Computing, Vienna, Austria.

Ripoll J., Urban L., \& Bertin N. (2016). The potential of the MAGIC TOM parental accessions to explore the genetic variability in tomato acclimation to repeated cycles of water deficit and recovery. Frontiers in plant science, 6, 1172 .

Schindelin J., Arganda-Carreras I., Frise E., Kaynig V., Longair M., Pietzsch T., Preibisch S., Rueden C., Saalfeld S., Schmid B., Tinevez J.-Y., White D. J., Hartenstein V., Eliceiri K., Tomancak P. \& Cardona A. (2012). Fiji: an open-source platform for biological-image analysis.Nature methods, 9(7), 676-682

Schreiber S. G., Hacke U. G. \& Hamann A. (2015). Variation of xylem vessel diameters across a climate gradient: insight from a reciprocal transplant experiment with a widespread boreal tree. Functional Ecology, 29(11), 1392-1401.

Sharma A. K. (1956). Fixation of plant chromosomes. The Botanical Review, 22(10), 665-695. Spiegelman Z., Ham B. K., Zhang Z., Toal T. W., Brady S. M., Zheng Y., Fei Z., Lucas W. J. \& Wolf S. (2015). A tomato phloem-mobile protein regulates the shoot-to-root ratio by mediating the auxin response in distant organs. The Plant Journal, 83(5), 853-863.

Szymkowiak E. J. \& Irish E. E. (1999). Interactions between jointless and wild-type tomato tissues during development of the pedicel abscission zone and the inflorescence meristem. The Plant Cell,11(2), 159-175.

Tolivia D. \& Tolivia J. (1987). Fasga: a new polychromatic method for simultaneous and differential staining of plant tissues. Journal of Microscopy, 148(1), 113-117. 
Van As H. (2007). Intact plant MRI for the study of cell water relations, membrane permeability, cell-to-cell and long distance water transport. Journal of Experimental Botany, 58(4), 743-756.

Van As H., Scheenen T. \& Vergeldt F. J. (2009). MRI of intact plants.Photosynthesis Research, 102(2-3), 213.

Van Bel A. J. E. (1990). Xylem-phloem exchange via the rays: the undervalued route of transport. Journal of Experimental Botany,41(6), 631-644.

Van Ieperen W., Volkov V. S. \& Van Meeteren U. (2003). Distribution of xylem hydraulic resistance in fruiting truss of tomato influenced by water stress. Journal of Experimental Botany, 54(381), 317-324.

Windt C. W., Vergeldt F. J., De Jager P. A. \& Van As H., (2006) MRI of long-distance water transport: a comparison of the phloem and xylem flow characteristics and dynamics in poplar, castor bean, tomato and tobacco.Plant, Cell \& Environment, 29(9), 1715-1729.

Windt C. W., Gerkema E. \& Van As H. (2009). Most water in the tomato truss is imported through the xylem, not the phloem: a nuclear magnetic resonance flow imaging study. Plant Physiology, 151(2), 830-842.

Figure 1: Transversal histological section of (A) a tomato fruit pedicel (fruit side or AZf) and (B) stem. Genotype: WVA106. FASGA stained. Section thickness: $60 \mu \mathrm{m}$. The yellow lines correspond to the segmentation of the different tissue types. Segmentation was done using a plugin (PHIV_Tomate_toolset) developed on Fiji allowing to measure the area of each segmented histological zone. For (A), scale bar: 1 $\mathrm{cm}$. For (B), scale bar : $2 \mathrm{~cm}$.

Figure 2: Histological sections (A and $\mathrm{B})$ and proportions of tissues $(\mathrm{C}$ and $\mathrm{D})$ along the tomato pedicel of Heinz wild-type (A and C) and Heinz Jointless (B and D) genotype. (A, B) Left: fruit side of the pedicel (AZf); middle: actual (wild type) or theoretical (jointless: at the level of the pedicel curvature) abscission zone (AZ); right: stem side of the pedicel (AZs). (C, D) The different shades represent the different tissue types (see label box). $\mathrm{N}=2$ to 8 fruits per genotypes. Error bars represent the standard deviation. Stars indicate significance differences between the proportion of tissue in the AZs and the AZf: ${ }^{*} \mathrm{p}<0.05,{ }^{* *} \mathrm{p}<0.01$. Transverse sections (thickness: $60 \mu \mathrm{m}$ ) were stained by FASGA. Scale bar $=1 \mathrm{~mm}$.

Figure 3: Influence of the genotype on pedicel and fruit growth. (A) Pedicel diameter normalized plant by plant by the the mean value of diameter at the plateau as a function of the fruit age normalized by the age at maturity. Each point represents one plant at a given time. A segmented model was fitted to experimental data (red dotted line), pooling all genotypes together and the break-point of this model corresponds to the time when the pedicel stopped growing. (B) Fruit diameter normalized plant by plant by the final diameter as a function of the normalized age of the fruit. Each curve represents the Gompertz model fitted to experimental data for each individual genotype. For (A) and (B) the different colors represent the different genotypes and the black dotted line indicates the breakpoint for the pedicel diameter. $\mathrm{N}=1$ to 5 fruits per genotype.

Figure 4: Fruit and pedicel traits depending on genotypes: (A) Final fruit fresh mass in g. (B) Final fruit dry matter content in g 100 g-1. (C) Final pedicel diameter in mm. (D) Area of the conductive tissues in the pedicel section in $\mu^{2}$. (E) Mean area proportion of the tissues in the pedicel section. The different shades represent the different tissues (see label box). For (A), (B), (C), (D) and (E), genotypes are ranked from the smaller to the larger genotype in terms of fresh mass, from left to right. Three categories of fruit size are indicated: small fruits $(\mathrm{FW}<15 \mathrm{~g})$, large fruits $(\mathrm{FW}>100 \mathrm{~g})$ and medium fruits between. $\mathrm{N}=2$ to 8 fruits per genotype.

Figure 5: Principal Component Analysis (PCA) of the genetic variations in fruit and pedicel traits. Each point represents the barycenter of the genotype, with error bar representing the standard deviation, and each color represents a genotype (see label box). Variables are scaled to unit variance and highly correlated variables have been removed $(>0.85)$. Small fruit: $\mathrm{FW}<15 \mathrm{~g}$, large fruits $>100 \mathrm{~g}$ and medium fruits between. $\mathrm{N}=2$ to 8 fruits per genotypes. 
Figure 6: Influence of water stress treatments on fruit and pedicel traits: (A) Final fruit fresh mass in g. (B) Final dry matter content in the fruit in g $100 \mathrm{~g}-1$. (C) Final pedicel diameter in mm. (D) Area of tissues in the pedicel section in $\mu \mathrm{m}^{2}$. (E) Mean area proportions of tissues in the pedicel section. The different shades represent the different tissues (see legend). Early water deficit (WD) occurred 15 days before truss anthesis, and late WD occurred 4 days after the truss anthesis. Light WD means a reduction of $50 \%$ of the well-watered control and severe WD means a reduction of $65 \%$ of the control. $\mathrm{N}=3$ to 9 fruits per treatment. Stars indicate significance differences between the proportion of the tissue in the corresponding treatment and the control treatment: ${ }^{*} \mathrm{p}<0.05,{ }^{* *} \mathrm{p}<0.01$.

Figure 7: Measured and simulated evolution of fruit dry mass (red), fresh mass (black) and dry matter content (green) of WVA106. Symbols are experimental measurements and solid lines represent the simulations under control condition. (A) Simulation of the genotypic variability with the Virtual fruit model. The dotted lines represent the simulations with a $10 \%$ reduction of Lx1 and Lp1, two model parameters that represent respectively the xylem and phloem conductance in the pedicel. The dashed lines represent the simulations with a $350 \%$ increase of Lx1 and Lp1. (B) Simulation of three water deficit scenarios. The dotted lines represent the simulations for the early light WD, with a $23 \%$ decrease of Lx1 and a $36 \%$ decrease of Lp1. The dashed lines represent the simulations for the early severe WD, with a $54 \%$ decrease of Lx1 and Lp1. The dashed and dotted lines represent the simulations for the late severe WD, with a $28 \%$ decrease of Lx1 and a $27 \%$ decrease of Lp1. For (A) and (B), all the other parameters remain unchanged. The pedicel boundary conditions in the model, ie the stem water potential and the sugar concentration in the phloem, varied respectively, from -2 bar at night to -4.6 bar at midday and 0.5 moles of sugar $\mathrm{dm}^{-3}$ sap.

Figure 8: Quantification of water flow in tomato stem by MRI: (A) Inflow MRI image of a tomato transversal stem section at the bottom of the main stem, under the first truss, in full-watered conditions.. Repetition time: $1200 \mathrm{~ms}$, slice thickness: $0.5 \mathrm{~mm}$ and echo time: $12 \mathrm{~ms}$. The white area represents a high flip-flop signal, corresponding to the active vessels evidenced by flow-MRI measurements. Systematic FF experiments were performed at different repetition times in order to determine the mean flux velocity. The time duration of the flow-MRI experiments depended on the repetition time and lasted from 4 min to 40 minutes, giving a total FF experiments time of 3 hours. (B) Histological FASGA stained slices of the same part of the plant. Thickness : $100 \mu \mathrm{m}$. Scale bar: $2 \mathrm{~mm}$. (C) Flip-flop signal as a function of the repetition time in second. The stars represent the measured FF signal at different repetition times and the red curve its best fit with the following fitting parameters : T1 $=1000 \mathrm{~ms}$, active area of $1.8 \mathrm{~mm}^{2}$ and mean velocity of $0.469 \mathrm{~mm} \mathrm{~s}^{-1}$. (D) Distribution of vessel diameters in $\mu \mathrm{m}$. In green: xylem vessels measured on the histological slice. In red: active xylem vessels estimated on the MRI image. Due to the lower resolution of MRI, the size of the largest vessels was slightly overestimated compared to the histological measurement.

\section{Hosted file}

Figures.pdf available at https://authorea.com/users/319610/articles/449332-structuraland-functional-contributions-of-conducting-tissues-to-genotypic-and-environmental-

variations-of-tomato-fruit-mass 